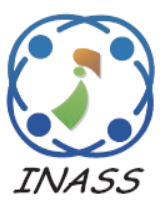

\title{
Applying Reversible Data Hiding for Medical Images in Hybrid Domain Using Haar and Modified Histogram
}

\author{
Vanmathi Chandrasekaran ${ }^{1 *} \quad$ Prabu Sevugan $^{2}$ \\ ${ }^{1}$ School of Information Technology and Engineering, ${ }^{2}$ School of Computer Sciences and Engineering \\ VIT University, Vellore, India \\ * Corresponding author’s Email: vaanmathiengr@gmail.com
}

\begin{abstract}
In this paper a reversible data hiding (RDH) algorithm for medical images is proposed. This algorithm specifically based on histogram modification in hybrid domain. The idea of this algorithm is that a histogram is created from the differences between each pixel and its neighbours. A 2D DWT haar transform is performed to convert cover image into a transform domain to select appropriate frequencies for embedding the payload. The selected DWT coefficients bit planes are used to carry the secret message. The experimental result demonstrates that our proposed method outperforms the traditional state of art methods in terms Peak Signal to Noise Ratio (PSNR), Structural Similarity Index (SSI) and the algorithm guarantees the reversibility of the host image. The proposed method avoids overflow and underflow of the pixel values and achieves highest embedding capacity.
\end{abstract}

Keywords: Reversible data hiding, Steganography, Haar wavelet, Histogram modification.

\section{Introduction}

Securing information transmitted over the Internet has turned into a basic issue driven by the advance in information digitalization and correspondences organizing over the previous decade. Data hiding technique aims to hide the secret information into the carrier without affecting. Cryptography, Steganography and watermarking are the three major techniques of data hiding. Cryptography encrypts the secret data ad reveals its existence. Steganography hides the secret data into the cover such as image, audio or video which makes difficult to identify the original cover and the cover containing the secret information. The result of data hiding introduces distortion in the cover signal. Watermarking also does the same thing, but it is used for copyright protection and its focus on robustness, whereas steganography focus on undetectably and capacity. Reversible data hiding $(\mathrm{RDH})$ is a technique where the carrier signal is recovered listlessly after the secret message is extracted. RDH is attracted by many researchers and widely used in forensics, military imagery and medical imagery where no distortion is allowed in the carrier signal. It is additionally confident that the first substance ought to be recuperated with no blunder after picture decoding and message extraction at beneficiary side. This exhibits a common-sense plan fulfilling the previously mentioned necessities. A substance proprietor encodes the first picture utilizing an encryption key, and an information hider can implant extra information into the scrambled picture utilizing an information concealing key however he doesn't know the first substance.

A variety of data hiding methods [1-3] has been surveyed and given in the literature such as steganography and watermarking however these techniques damages the carrier while hiding the data. Steganography guarantees the confidentiality and security of the secret data without confronting the attention to the nasty attackers. Data hiding is divided into two categories based on the recovery of the original cover object: reversible data hiding and irreversible data hiding. The benefit of reversible data hiding is that the payload is high and 
it modifies the cover image which cannot be get better after extracting the secret.

Secret information can be hidden in three domains: spatial, frequency and compressed. In spatial domain, the data are hidden on the direct pixel values of the cover image. In the frequency domain, the secret message is hidden by modifying the frequency coefficients of the cover image in the frequency domain. The cover image is compressed before using it for data hiding in compressed domain. This paper presents a data hiding scheme based on transform domain.

RDH methods fall into 4 types. The first type uses compression framework which is introduced by Fridrich[ 4], first extracts the LSB of the cover image pixel and compresses it. The LSB of the cover image is appended into the secret message before encryption. However, the payload is pretty low. The second method is difference expansion (DE) in which the pixel groups are expanded based on the differences for example multiplied by two. The pixel group which has all zero differences of LSB can be used for secret message replacement. The location map is not required for the decryption. The third RDH method is based on histogram shifting [5] where the histogram of the image is modified to embed the secret message. The capacity is depending on the highest histogram value. The fourth method is based on integer transform [6-8], in this the image is represented in integer transform domain for data hiding. Out of these methods integers transform methods yields enhanced data capacity. The proposed method provides reversibility of the source image with high embedding rate and high imperceptibility by avoiding overflow and underflow of the pixel values. In our method, data is hidden in wavelet domain and the auxiliary data required for recovery of the original image is done using histogram modification technique.

The performance of the stego system is measured by using three metrics they are payload, imperceptibility and robustness. Spatial domain data hiding results more payload and imperceptibility but less robustness. Transform domain techniques provides more imperceptibility and robustness with acceptable payload. The paper is organized as Section 2 the related works and in Section 3 Proposed work embedding and extraction process. Section 4 experimental results and analysis followed by a conclusion in section 5 .

\section{Related work}

Tian et al. [9] proposed RDH scheme based on DE. The source image is divided into pixel pairs and it is embedded with 1 bit of secret data. The pixel pairs ensure non-existence of overflow and underflow. To identify the pairs modified during the embedding, the location of the compressed pairs is stored and appended to the payload. This method is suitable for lesser payload and it can be increased if multiple LSBs are used.

Y Hu et al. [10] presented an efficient location map to reduce the size of the location data and thereby increasing the payload. However, authors have not achieved the better accuracy for the algorithm. Yang et al. [11] proposed reversible watermarking in compressed domain. The adjacent block values are used to encode the current block with additional requirement of the flag bits. The VQ table is modified to achieve better reversibility. The usage of flag bits increased the stego image load thereby reduces the quality.

Kalker and Williams [12] designed a RDH model for rate distribution. The upper bound problem for embedded payload is solved by formulating a rate distribution function and the test comes about gives traded off outcomes. They proved the fee-distortion bounds of $\mathrm{RDH}$ for reminiscence much less covers. Alattar et al. [13] used three to four pixels for data embedding using DE which increases the data capacity from 0.5 to $0.75 \mathrm{bpp}$. The location map is size is equal to $1 / 4$ th of the cover image size. Tai et al. [14] divided the image into smaller blocks and the peak values of the histogram in each block are identified to embed the secret message. However the authors did not focused on the prediction errors which lead to less accuracy in reversibility.

Lee et al. [15] used the neighbours mean values to predict the histograms which are suitable for data embedding with lesser embedding rate. Yang et al [16] used interleaving predictions to guarantee a histogram and the data is embedded around the peak values. This method increased the robustness of the algorithm. But the data embedding is performed in spatial domain, it reduces visual perception.

Wien Hong et al. [17] presented RDH for encrypted images. The image is divided into blocks and each block holds one bit of the secret data for flipping three LSB of the predefine pixel block. The error rate of the extracted secret data bits is reduced by using the side match method. This method results overflow and underflow of the pixel values results increases the complexity in retrieval of the secret data and source image.

Further, with a purpose to keep away from hackers or attackers duplicated or revised medical 
facts thru the net and to preserve the clinical image first-class for accurate diagnosis, reversible data hiding plays a critical role in medical image processing. Yuling et al. [18] portioned the image into smooth region and non-smooth region and applied Diamond encoding method for data embedding to achieve high data capacity. Besides that the advantage shrinks that is the quality of the stego image reduced when the data size is increased. Hao et al [19] proposed ROI "Region of Interest" based RDH which embeds secret information into non-ROI by means of an irreversible hiding technique and in ROIs through a reversible image hiding method. During embedding process a small visual distortion is produced and also it cannot completely restore the image back to the original.

From the literature on RDH the quality of the stego image is measured by PSNR. The histogram modification used in the proposed work improves the inconsistency in image quality with human visual perception and also for reversibility. Hiding data in transform domain provides robustness to the algorithm. Most of the existing methods concentrate on payload and confidentiality, in conventional transform methods not guarantees the reversibility of the original image. To overcome the problem integer to integer transform is applied in the proposed work. The mapping is performed on integer to integer values both in reverse and forward transform.

\section{Proposed work}

\subsection{RDH in transform domain}

Wavelet transform converts the Image from spatial domain to transform domain [20], normally a wavelet conversion is based on the floating-point operation. When we apply this technique specifically to implant messages into a picture for reversible data hiding, a truncation blunder might be experienced, prompting to the disappointment of the message extraction and image restoration. To avoid this problem, the invertible integer to integer wavelet transform was proposed by Daubechies [21] converts an image into frequency domain without data loss so that it making it suitable for reversible data hiding. Discrete wavelet transform decomposes the image into four sub bands: LL, HL, LH and HH. LL is the approximate coefficient of the image, HL, LH is horizontal and vertical coefficients respectively and $\mathrm{HH}$ is the diagonal detailed feature of the image [22]. Fig 1 shows the IWT processing levels in 2 dimensional. In order to decompose the image further 2D wavelet transform is recursively applied to the approximation coefficients. Fig 2 depicts two level HDWT decomposition representations of Lena image is shown.

\subsection{Histogram modification}

Histogram modification technique is one of the remarkable works of $\mathrm{RDH}$ [23] where the histograms of the peak points are used for data embedding. Before using the peak points the histogram bins between the zero point and the peak points are shifted. Lee et al. [24] Proposed and method for constructing a sharper histogram by using the difference histogram of the image. Afterwards Tsai et al. [25] proposed an algorithm using the correlation between the pixels is considered while constructing the histogram. Pan et al. [26] divided the image into blocks, histogram is constructed for each block and then the differences between the pixels are computed using the peak point in the selected block. In our proposed method, the histogram modification is used to embed the auxiliary data required for reversibility of the original image. The main idea of the work is to implement $\mathrm{RDH}$ using integer transform and histogram modification providing low distortion with high payload.

The proposed method uses hybrid domain to perform reversible data hiding. The secret data is embedded in transform domain using haar transform and the auxiliary information required for reversibility of the host image is embedded in spatial domain using histogram modification technique. The auxiliary data carries the host image information to recover the original image from the stego image without any loss. Fig 3 shows the embedding steps of RDH. The following depicts the sequence of steps involved in auxiliary data embedding.

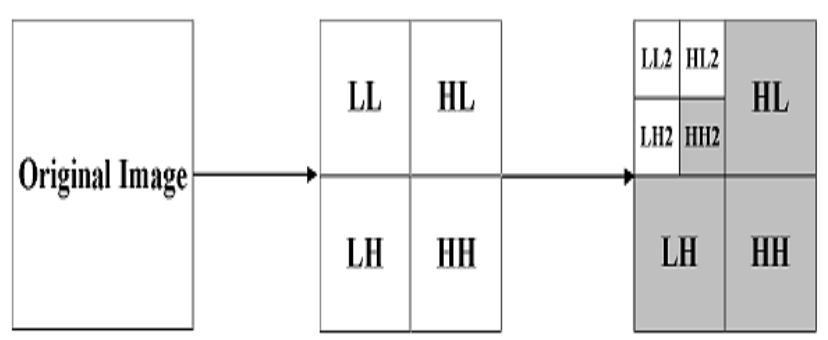

Figure. 1 Decomposition of 2 level IDWT 


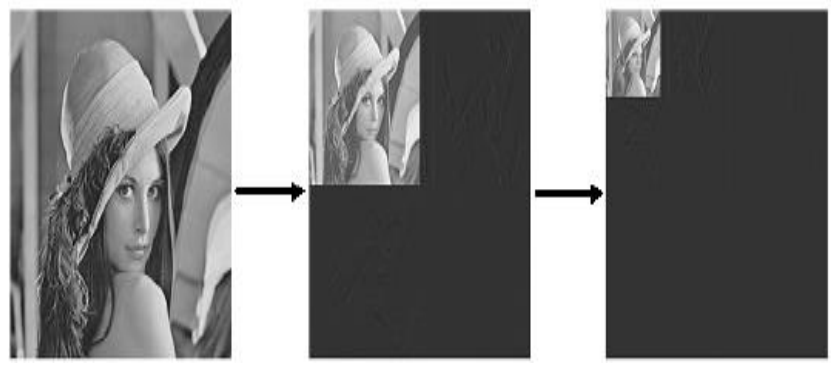

Figure. 2 Two level HDWT representation of Lena image

1. Scan the image and Calculate pixel difference $D_{i}$ between $C_{i}$ and $C_{i}-1$

$$
D i=\left\{\begin{array}{cc}
C i & \text { if } i=0 \\
|C i-1| & \text { otherwise }
\end{array}\right.
$$

2. Find the peak point PP value from $D_{i}$. If $D_{i}>P P$ shift $C_{i}$ by 1

$$
S i=\left\{\begin{array}{cl}
C i, & \text { if } i=0 \text { or } D i<P P \\
C i+1, & \text { if } D i>P P \text { and } C i \geq C i-1 \\
C i-1 & \text { if } D i>P P \text { and } C i<C i-1
\end{array}\right.
$$

Where $S_{i}$ is the stego image pixel value

3. If $D_{i}=P P$ change value of $C_{i}$ according to the auxiliary data $A D$ bit $x$

$$
S i=\left\{\begin{array}{l}
C i+x \text { if } D i=P P \text { and } C i \geq C i-1 \\
C i-x, \text { if } D i=P P \text { and } C i<C i-1
\end{array}\right.
$$

Data extraction at the receiver side from the stego image pixel is by using the following

$$
x=\left\{\begin{array}{c}
0, \text { if }|S i-S i-1|=P P \\
1, \text { if }|S i-S i-1|=P P+1
\end{array}\right.
$$

The original pixel value is restored by

$$
\begin{aligned}
& C i \\
& =\left\{\begin{array}{l}
S i+1, \text { if }|S i-S i-1|>P P \text { and } S i<C i-1 \\
S i-1, \text { if }|S i-S i-1|>P P \text { and } S i>C i-1
\end{array}\right.
\end{aligned}
$$

\subsection{Data embedding algorithm}

Step 1: Input the host image Ic

Step 2: Pre-process the image Ic

Step 3: Transform the image using Haar integer to integer transform
Step 4: Scan the high and middle frequencies in zig zag pattern and embed the secret data.

Step 5: The replaced frequency values are stored as an auxillary data (AD)

Step 6: Apply Inverse Haar transform to get Intermediate Haar Transform Image (IHI)

Step 7: AD is embedding into IHI using Histogram Modification to get the stego image.

\subsection{Data extraction algorithm}

Step 1: Input the stego image

Step 2: Apply histogram recovery to retrieve auxiliary data from the stego image.

Step 3: Convert the image to transform domain image using Haar transform.

Step 4: Extract the secret data from the middle and high frequency components of the transform with the help of auxiliary data.

Step 5: Apply inverse integer transform

Step 6: Recover the host image without any loss.

From the above algorithm, the following points helps to make our proposed method performs better. First the scanning order zig zag of the coefficients in transform domain. Second it achieves good results since the pixel pair coordination is used and finally the option for selecting the PP is determined by the applications and based on the histogram characteristics. To get high image quality the PP should be chosen for less bin shifts.

\section{Experimental results and analysis}

The proposed algorithm is tested in MATLAB R2010 with the sample images. The experimental results are calculated for PSNR and SSIM values of the existing methods and the proposed method. The embedding rate [27-29] methods are virtually not changed. The authors used medical image smooth area as a higher priority to hide the secret data, the non-smooth area is used lesser compared to smooth region. So, the stego images are not visual to human visual system. Wu et al uses two highest bins of the image histogram for gray images and used image enhancement to increase the contrast of the image background. But the proposed method restores the 


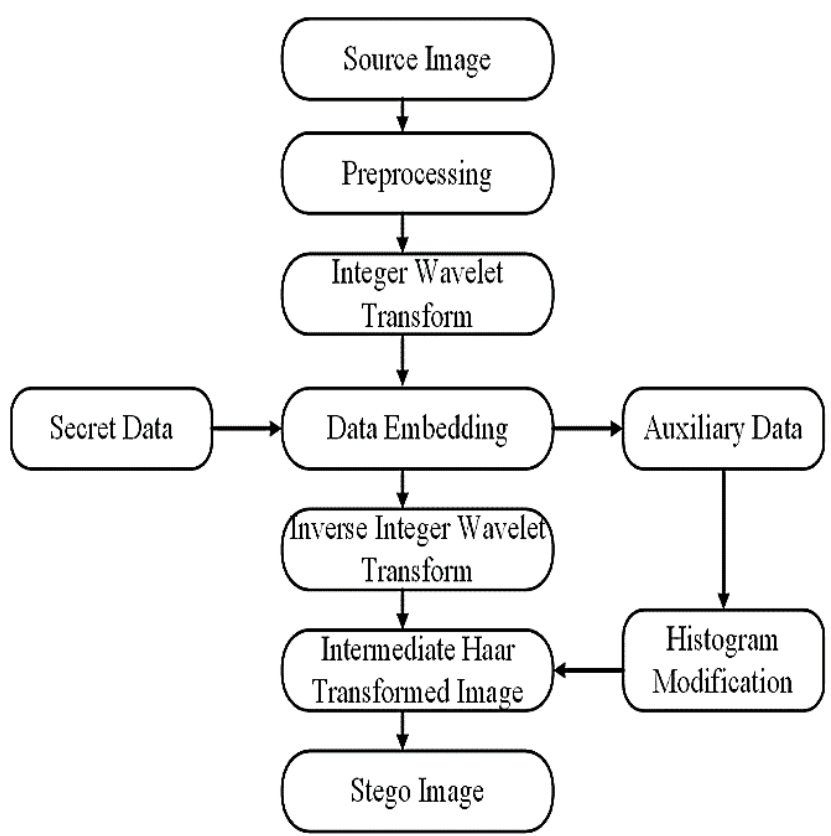

Figure. 3 Data embedding

information of the auxiliary data as well as the secret data. From the tabular values, it is clear that the proposed method gives compromised results.

\section{Mean Square Error (MSE)}

MSE is the square of the difference between pixel values of the stego image and cover image divided by the size of the image. The following formula gives an MSE value between $X$ and $Y$ image of size $M x N$

$$
\mathrm{MSE}=\frac{1}{N x M} \sum_{i=0}^{N-1} \sum_{j=0}^{M-1}[X(i, j)-Y(i, j)]^{2}
$$

\section{Peak Signal to Noise Ratio (PSNR)}

PSNR is the universally used metric to discover the distortion between the stego image and the cover image quality. Higher the PSNR value results the better image quality and the following gives the formula for PSNR calculation.

$$
\mathrm{PSNR}=20 \log _{10}\left(\frac{\mathrm{MAXf}}{\sqrt{\mathrm{MSE}}}\right)
$$

Where $M A X_{f}-$ Maximum pixel value and MSE-

Mean Square Error

\section{Universal Image Quality Index (QI)}

In 2002, Wang developed a measure based on three factors loss of correlation, luminance distortion and contrast distortion. The range of $Q I$ is between [-1,1]. The value of $Q I$ is normalized to 1 in case of similar images.

$$
Q I=\frac{\sigma x y \bar{x} \bar{y}}{\left(\sigma x^{2}+\rho y^{2}\right)\left[\bar{x}^{2}+\bar{y}^{2}\right]}
$$

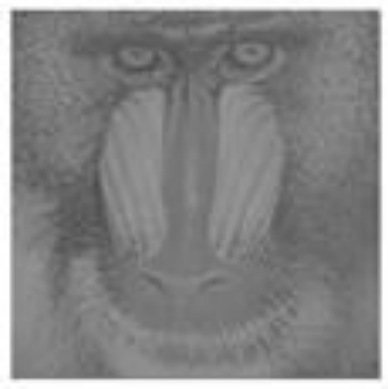

(a)

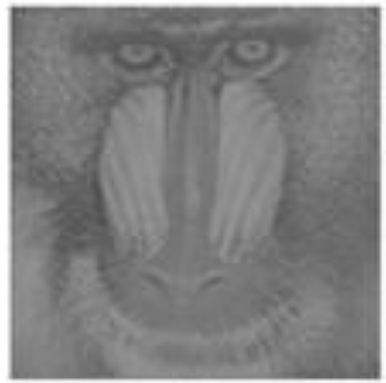

(b)
Figure. 4 Test Image: (a) Baboon cover image and (b) Stego image

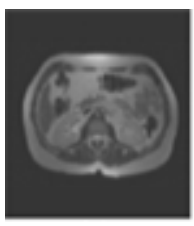

(a)

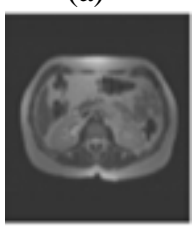

(e)

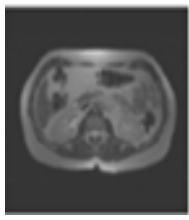

(i)

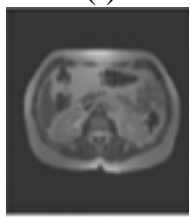

(m)

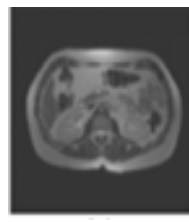

(b)

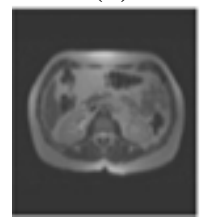

(f)

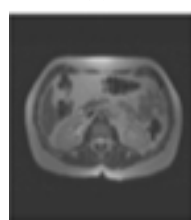

(j)

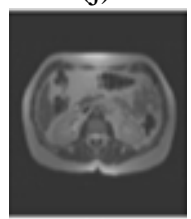

(n)

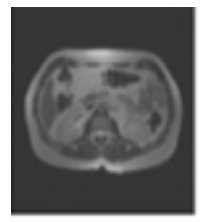

(c)

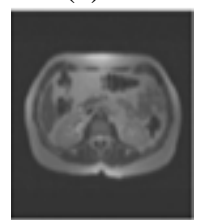

$(\mathrm{g})$

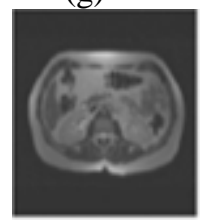

(k)

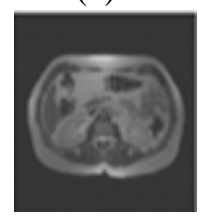

(o)

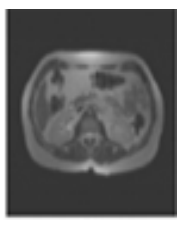

(d)

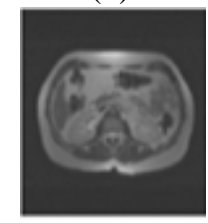

(h)

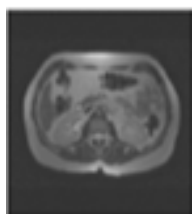

(1)

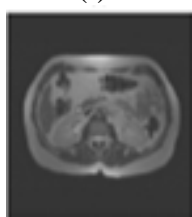

(p)
Figure. 5 Hepatitis marked Margo interior images in $0.1 \mathrm{bpp}, 0.3 \mathrm{bpp}, 0.6 \mathrm{bpp}$ and $0.8 \mathrm{bpp}$ respectively. 


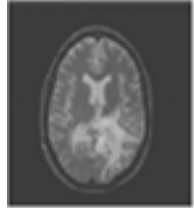

(a)

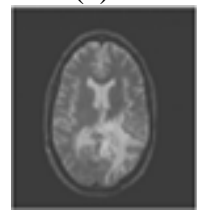

(e)

(i)

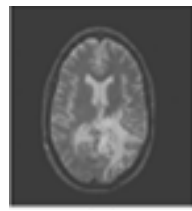

(m)

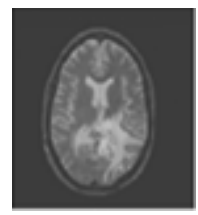

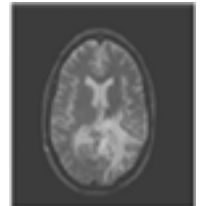

(b)

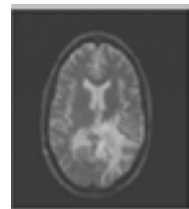

(f)

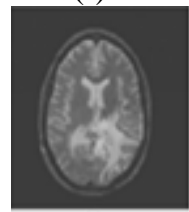

(j)

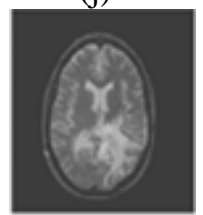

(n)

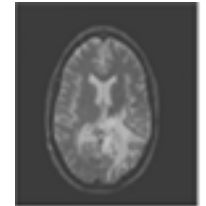

(c)

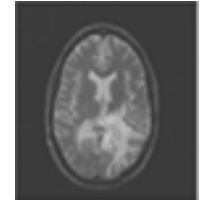

(g)

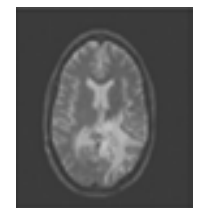

(k)

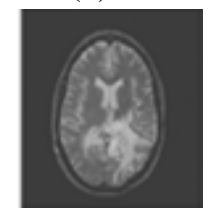

(o)

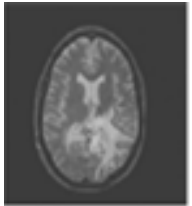

(d)

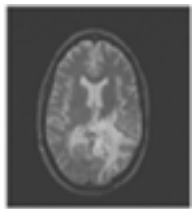

(h)

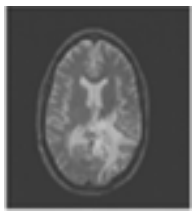

(1)

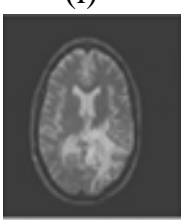

(p)
Figure. 6 Brain marked image with various range of bits per pixel $0.1 \mathrm{bpp}, 0.3 \mathrm{bpp}, 0.6 \mathrm{bpp}$ and $0.8 \mathrm{bpp}$ respectively.

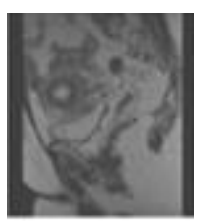

(a)

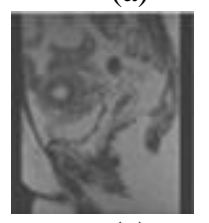

(e)

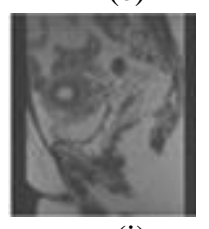

(i)

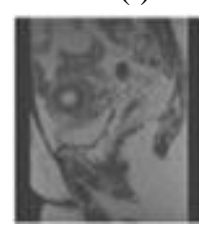

(m)

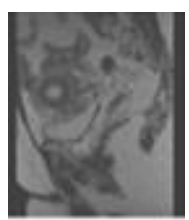

(b)

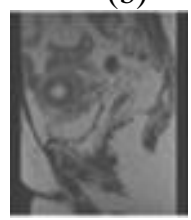

(f)

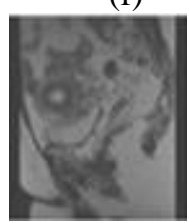

(j)

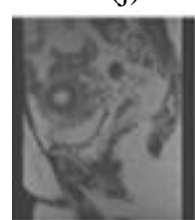

(n)

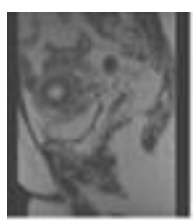

(c)

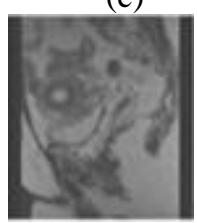

(g)

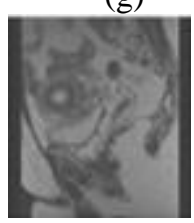

(k)

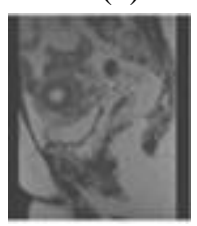

(o)

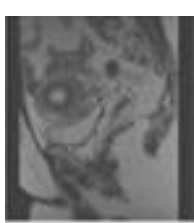

(d)

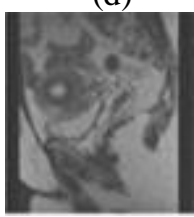

(h)

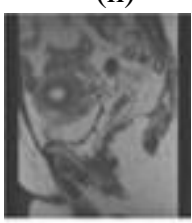

(1)

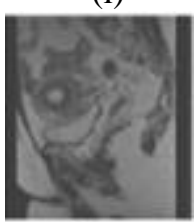

(p)
Figure. 7 Pelvic Cavity images with $0.1 \mathrm{bpp}, 0.3 \mathrm{bpp}$, $0.6 \mathrm{bpp}$ and $0.8 \mathrm{bpp}$ respectively.
Table 1. Comparative performance of Hepatitis marked images with Sachnev et al [25], Wu et al. [26] and Gao et

\begin{tabular}{|c|c|c|c|c|}
\hline $\begin{array}{l}\text { RDH } \\
\text { Method }\end{array}$ & Fig No & BPP & PSNR & SSIM \\
\hline \multirow{4}{*}{$\begin{array}{l}\text { Sachnev } \\
\text { et al. }\end{array}$} & Fig 5 a & 0.1 & 60.9414 & 0.9993 \\
\hline & Fig $5 \mathrm{~b}$ & 0.3 & 49.7934 & 0.9985 \\
\hline & Fig $5 \mathrm{c}$ & 0.6 & 48.4432 & 0.9948 \\
\hline & Fig $5 \mathrm{~d}$ & 0.8 & 43.3425 & 0.9900 \\
\hline \multirow[t]{4}{*}{ Wu et al. } & Fig 5 e & 0.1 & 61.1395 & 0.9996 \\
\hline & Fig $5 \mathrm{f}$ & 0.3 & 50.7932 & 0.9980 \\
\hline & Fig $5 \mathrm{~g}$ & 0.6 & 49.4439 & 0.9948 \\
\hline & Fig $5 \mathrm{~h}$ & 0.8 & 44.3429 & 0.9900 \\
\hline \multirow[t]{4}{*}{ Gao et al } & Fig 5 i & 0.1 & 61.3456 & 0.9942 \\
\hline & Fig $5 \mathrm{j}$ & 0.3 & 51.6541 & 0.9922 \\
\hline & Fig $5 \mathrm{k}$ & 0.6 & 49.5639 & 0.9956 \\
\hline & Fig 51 & 0.8 & 44.6231 & 0.9976 \\
\hline \multirow{4}{*}{$\begin{array}{l}\text { Proposed } \\
\text { Method }\end{array}$} & Fig $5 \mathrm{~m}$ & 0.1 & 62.5641 & 0.9991 \\
\hline & Fig $5 n$ & 0.3 & 52.8515 & 0.9898 \\
\hline & Fig 5 o & 0.6 & 50.9214 & 0.9994 \\
\hline & Fig $5 p$ & 0.8 & 45.8586 & 0.9991 \\
\hline
\end{tabular}

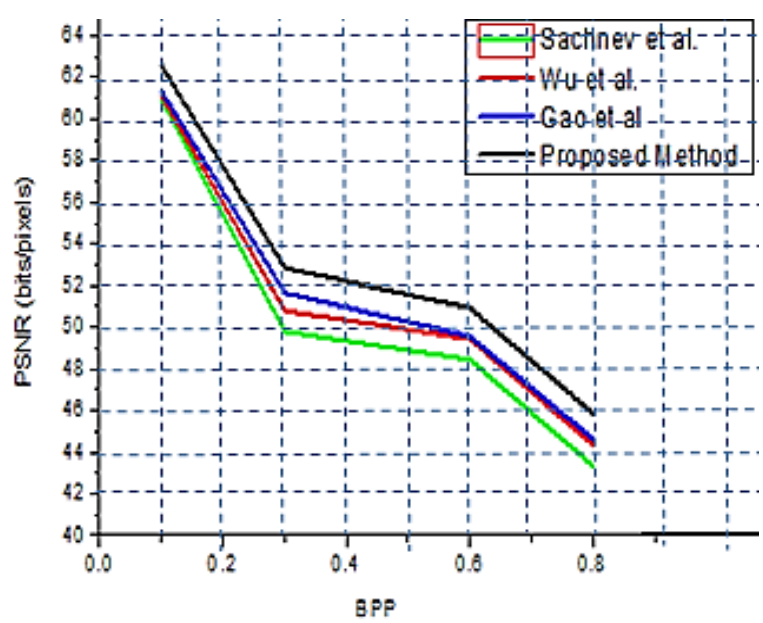

Figure. 8 Performance comparisons for Hepatitis Marked Medical Images

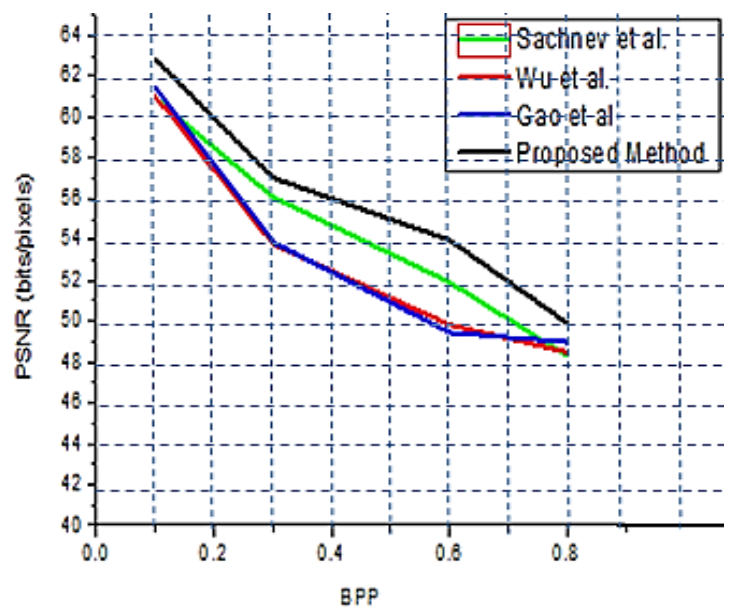

Figure. 9 Performance Comparisons for Brain Marked Medical Images 
Table 2. Comparative performance of brain marked images with Sachnev et al [25], Wu et al. [26] and Gao et

\begin{tabular}{|c|c|c|c|c|}
\hline \multirow{2}{*}{$\begin{array}{c}\text { RDH } \\
\text { method }\end{array}$} & Fig No & Bpp & PSNR & SSIM \\
\hline Sachnev & & & & \\
\hline \multirow{4}{*}{ et al. } & Fig 6 a & 0.1 & 60.9275 & 0.9994 \\
\cline { 2 - 5 } & Fig 6 b & 0.3 & 56.0862 & 0.9981 \\
\cline { 2 - 5 } & Fig 6 c & 0.6 & 51.9114 & 0.9965 \\
\cline { 2 - 5 } & Fig 6 d & 0.8 & 48.3201 & 0.9880 \\
\hline Wu et al. & Fig 6 e & 0.1 & 61.0277 & 0.9996 \\
\cline { 2 - 5 } & Fig 6 f & 0.3 & 53.7873 & 0.9987 \\
\cline { 2 - 5 } & Fig 6 g & 0.6 & 49.8257 & 0.9972 \\
\cline { 2 - 5 } & Fig 6 h & 0.8 & 48.4978 & 0.9943 \\
\hline Gao et al & Fig 6 i & 0.1 & 61.4532 & 0.9967 \\
\cline { 2 - 5 } & Fig 6 j & 0.3 & 53.8732 & 0.9948 \\
\cline { 2 - 5 } & Fig 6 k & 0.6 & 49.4531 & 0.9965 \\
\cline { 2 - 5 } & Fig 6 1 & 0.8 & 48.9856 & 0.9945 \\
\hline Proposed & Fig 6 m & 0.1 & 62.8465 & 0.9995 \\
\cline { 2 - 5 } Method & Fig 6 n & 0.3 & 57.0325 & 0.9998 \\
\cline { 2 - 5 } & Fig 6 o & 0.6 & 53.9809 & 0.9999 \\
\cline { 2 - 5 } & Fig 6 p & 0.8 & 49.9156 & 0.9997 \\
\hline
\end{tabular}

Table 3. Comparative performance of pelvic cavity marked images with [25], [26] and [27]

\begin{tabular}{|c|c|c|c|c|}
\hline $\begin{array}{c}\text { RDH } \\
\text { method }\end{array}$ & Fig No & Bpp & PSNR & SSIM \\
\hline \multirow{4}{*}{$\begin{array}{l}\text { Sachnev } \\
\text { et al. }\end{array}$} & Fig 7 a & 0.1 & 60.2826 & 0.9995 \\
\hline & Fig 7 b & 0.3 & 49.3696 & 0.9953 \\
\hline & Fig 7 c & 0.6 & 46.9819 & 0.9744 \\
\hline & Fig $7 \mathrm{~d}$ & 0.8 & 41.9747 & 0.9518 \\
\hline \multirow[t]{4}{*}{ Wu et al. } & Fig 7 e & 0.1 & 57.0086 & 0.9996 \\
\hline & Fig $7 \mathrm{f}$ & 0.3 & 46.3418 & 0.9977 \\
\hline & Fig $7 \mathrm{~g}$ & 0.6 & 44.9762 & 0.9717 \\
\hline & Fig $7 \mathrm{~h}$ & 0.8 & 36.3102 & 0.9544 \\
\hline \multirow[t]{4}{*}{ Gao et al } & Fig $7 \mathrm{i}$ & 0.1 & 58.8761 & 0.9689 \\
\hline & Fig $7 \mathrm{j}$ & 0.3 & 46.9865 & 0.9699 \\
\hline & Fig $7 \mathrm{k}$ & 0.6 & 45.7231 & 0.9776 \\
\hline & Fig 71 & 0.8 & 37.7633 & 0.9865 \\
\hline \multirow{4}{*}{$\begin{array}{l}\text { Proposed } \\
\text { Method }\end{array}$} & Fig $7 \mathrm{~m}$ & 0.1 & 62.8784 & 0.9945 \\
\hline & Fig $7 \mathrm{n}$ & 0.3 & 50.8712 & 0.9987 \\
\hline & Fig 7 o & 0.6 & 47.9819 & 0.9991 \\
\hline & Fig $7 \mathrm{p}$ & 0.8 & 42.9874 & 0.9990 \\
\hline
\end{tabular}

\subsection{Comparison with existing work}

In order to illustrate the proposed method characteristic first it is tested on baboon image with Sachnev et al. [25] with the PSNR $=39.8418 \mathrm{~dB}$, SSIM=0.9666, RCE=0.4956 shown in Fig 4. Fig 5, Fig 6 and Fig 7 shows the impact of embedding $0.1 \mathrm{bpp}, 0.3 \mathrm{bpp}, 0.6 \mathrm{bpp}$ and $0.8 \mathrm{bpp}$ on the medical images and represents the visual distortion induced in the image. Steganography in transform domain is used by the existing work is considered for comparing the results. Table 1, table 2 and table 3gives the comparative results with Sachnev et al. [25], Wu et al. [26] and Gao et al. [27] for hepatitis, pelvic cavity and brain marked images. The test has been carried out with various images and the output also shown.

\subsection{Performance analysis}

The payloads with various sizes are tested for the proposed algorithm with various types of medical images. It is noticed that the image quality is decreased when the payload increases. The results obtained recites that the proposed method achieves the good and better PSNR value. On the stego image noise introduced by the secret data is considerably reduced after the payload embedding. Further the result confirms that payload increased not affecting the quality of the stego image and also the cover image is recovered properly. The Fig 8, Fig 9 and Fig. 10 gives the graphical representation of the proposed work with payload verses PSNR for the various test medical images for the various existing RDH methods. The result parameters are calculated from the mean results of sixteen medical images with three different types. We notice from the graph that the PSNR values are slightly increased when the bit rate up to 0.6. There is a significant change in the PSNR values when it increased to $0.8 \mathrm{bpp}$. The usage of histogram modification in the proposed method gives better quality stego image. This method is successful in producing high data embedding rate with reversibility.

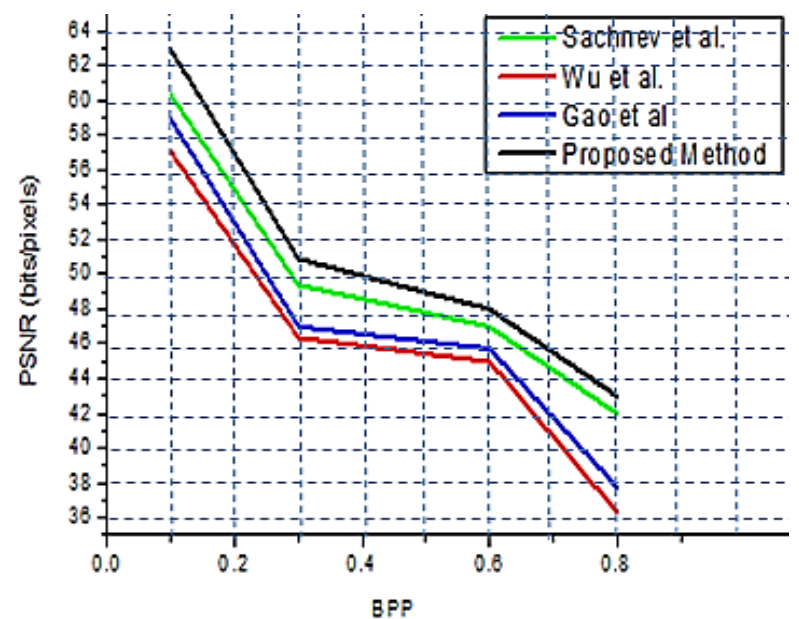

Figure. 10 Performance Comparisons for Pelvic Cavity Medical Images. 


\section{Conclusion}

A new Reversible data hiding based on histogram modification in hybrid domain was proposed in this paper which aims the medical images. Compared to the traditional histogram modification methods, our proposed method used the histogram based on the pixel difference between the neighbouring pixels. So, the modification of the pixel values in the stego image is more or less equal to the original image there by maintaining the good PSNR ratio. The proposed method achieves higher payload and better image quality since the data is hidden in transform domain. The retrieval of complete auxiliary information helps to recover the cover images without any loss. The strategies employed in our proposed method is scanning order of the pixel values in zig zag pattern and histogram modification techniques out performs compared to exiting $\mathrm{RDH}$ techniques. The experimented results of marked medical images demonstrate the proposed method has better image quality compared to similar RDH existing state of the art methods. The result also verifies the obtained PSRNR values are consistent with subjective visual perception. As a future work the embedding capacity of the data will be further considered for improvement.

\section{References}

[1] I. Cox, M. Miller, J. Bloom, J. Fridrich, and Kalker, Digital Watermarking and Steganography, Second Edition, Morgan Kaufmann Publishers, 2007.

[2] T. Mercuri, "The many colors of multimedia security", Communications of the ACM, Vol. 47, No. 12, pp. 25-29, 2004.

[3] P. Wayner, Disappearing cryptography, Morgan Kaufmann Publishers, San Francisco, CA, USA, 2002.

[4] J. Fridrich and M. Goljan, "Lossless data embedding for all image formats", In: Proc. of the Photonics West, Electronic Imaging, Security and Watermarking of Multimedia Contents, Vol. 4675, pp. 572 - 583, 2002

[5] J. Tian, "Reversible data embedding using a difference expansion", IEEE Transactions on Circuits and Systems for Video Technology, Vol. 13, No. 8, pp. 890-896, 2003.

[6] D. Thodi and J. Rodriguez, "Expansion embedding techniques for reversible watermarking," IEEE Transaction on Image Processing, Vol. 16, No. 3, pp. 721-730, 2007.

[7] Z. Ni, Y. Shi, N. Ansari, and S. Wei, "Reversible data hiding", IEEE Transactions
Circuits Systems Video Technology, Vol. 16, No. 3, pp. 354 - 362, 2006.

[8] P. Tsai, Y. C. Hu, and H. L. Yeh, "Reversible image hiding scheme using predictive coding and histogram shifting”, Signal Processing, Vol. 89, pp. 1129-1143, 2009.

[9] J. Tian, "Reversible data embedding using a difference expansion", IEEE Transactions on Circuits and Systems for Video Technology, Vol. 13, No. 8, pp. 831-841, 2003.

[10] Y. Hu, H. K. Lee, and J. Li, "DE-based reversible data hiding with improved overflow location map", IEEE Transactions on Circuits and Systems for Video Technology, Vol. 19, No. 2, pp. 250 - 260, 2009.

[11] C.H. Yang, M.H. Tsai, "Improving histogrambased reversible data hiding by interleaving predictions", IET Image Processing, Vol 4, No. 4, pp. 223 - 234, 2003.

[12] T. Kalker and F.M. Willems, "Capacity bounds and code constructions for reversible datahiding", In: Proc. of the 14th International Conference on Digital Signal Processing, Greece, pp. 71 -76, 2002.

[13] A. M. Alattar, "Reversible watermark using the difference expansion of a generalized integer transform", IEEE Transactions on Image Processing, Vol. 13, No. 8, pp. 1147 -1156, 2004.

[14] W.L. Tai, C.M. Yeh, C.C. Chang, "Reversible data hiding based on histogram modification of pixel differences", IEEE Transactions on Circuits Systems for Video Technology, Vol 19, No. 6, pp. 906 - 910, 2009.

[15] C.F. Lee, H.L. Chen, H.K. Tso, "Embedding capacity raising in reversible data hiding based on prediction of difference expansion", Journal of System Software, Vol. 83, No. 10, pp. 1864 1872, 2010.

[16] C.H. Yang, M.H. Tsai, "Improving histogrambased reversible data hiding by interleaving predictions", IET Image Processing, Vol. 4, No. 4, pp. 223-234, 2010.

[17] W. Hong, T.S. Chen, and W. Han-Yan, "An Improved Reversible Data Hiding in Encrypted Images Using Side Match", IEEE Signal Processing Letters, Vol. 19, No. 4, pp. 199-202, 2012.

[18] Y. Liu, X. Qu, G. Xin, "A ROI-based reversible data hiding scheme in encrypted medical images", Journal of Visual Communication and Image Representation, Vol. 39, pp. 51-57, 2016.

[19] H. Wu, J. Huang, Y. Shi, "A reversible data hiding method with contrast enhancement for 
medical images", Journal of Visual Communication and Image Representation, Vol. 31, pp. 146-153, 2015.

[20] C. Chang, C. Lin, C. Tseng and W. Tai, "Reversible hiding in DCT-based compressed images", Information Sciences, Vol. 177, No. 13, pp. 2768-2786, 2007.

[21] A. R. Calderbank, I. Daubechies, W. Sweldens, and B. L. Yeo, "Wavelet transforms that map integers to integers", Applied Computational Harmonics Analysis, Vol. 5, No. 3 , pp.332-369, 1998.

[22] A.N. Akansu and A. Haddad, Multiresolution Signal Decomposition, Transforms, Subbands, and Wavelets, Series in Telecommunications, Academic Press, 2001.

[23] Z. Ni, Y.Q. Shi, N. Ansari, W. Su, " Reversible data hiding", IEEE Transactions on Circuit and Systems for Video Technology, Vol. 16, No. 3, pp. $354-362,2006$.

[24] S.K. Lee, Y.H. Suh, and Y.S. Ho, "Reversible image authentication based on watermarking", Proceedings on IEEE ICME, pp. 1321 -1324, 2012.

[25] P. Tsai, Y.C. Hu, H.L. Yeh, "Reversible image hiding scheme using predictive coding and histogram shifting", Journal of Signal Processing, Vol. 89, No. 6, pp. 1129 -1143, 2009.

[26] Z.B. Pan, S. Hu, X.-X. Ma, L.F. Wang, "Reversible data hiding based on local histogram shifting with multilayer embedding", Journal of Visual Communication and Image Representation, Vol. 31, No. 64 -74, 2012.

[27] V. Sachnev, H.J. Kim, J. Nam, S. Suresh, Y.Q. Shi, "Reversible watermarking algorithm using sorting and prediction", IEEE Transactions on Circuits and Systems for Video Technology, Vol. 19, No. 7, pp. 989 - 999, 2009.

[28] H.T. Wu, J. Dugelay, Y.Q. Shi, "Reversible image data hiding with contrast enhancement", IEEE transactions on Signal Processing Letters, Vol. 22, No. 22, pp. 81 - 85, 2015.

[29] G.Y. Gao, Y.Q. Shi, "Reversible data hiding using controlled contrast enhancement and integer wavelet transform", IEEE transactions on Signal Processing Letters, Vol. 22, No. 11, pp. 2078-2082, 2015. 